\title{
Femtosecond Laser-Enabled TriBeam as a Platform for Analysis of Thermally- and Charge-Sensitive Materials
}

\author{
SJ Randolph ${ }^{1 *}$, R Geurts $^{2}, \mathrm{~J}_{\text {Wang }}{ }^{1}, \mathrm{~B}$ Winiarski ${ }^{3}$ and C Rue ${ }^{1}$ \\ 1. Thermo Fisher Scientific, Hillsboro, OR, USA. \\ 2. Thermo Fisher Scientific, Eindhoven, Netherlands. \\ 3. Thermo Fisher Scientific, Brno, Czech Republic. \\ * Corresponding author: steven.randolph@thermofisher.com
}

The challenges presented by beam-sensitive materials range from simple charging artifacts in imaging to actual beam-induced damage. One of the most common uses of focused ion beam (FIB) is to provide a high-resolution means of removing material deterministically so that the sample cross-section may be viewed in situ with an electron microscope (DualBeam ${ }^{\mathrm{TM}}$ FIB/SEM process). There are a few classes of materials/samples that present some unique challenges to FIB/SEM workflows such as highly nonconductive materials, damage/amorphization-prone samples, weakly-bound composite layers, and an abundance of polymeric materials. As such, there is a growing need for additional means of in situ material removal that may work in concert with FIB to address these challenging samples. Athermallyablative femtosecond (fs) laser technology has now reached the point of maturity that enables it to fill this gap.

Materials processed by fs-laser nearly universally demonstrate superior laser cut quality over thermallyablated surfaces, but neither can produce the final surface quality of FIB. However, the removal rates can be many orders of magnitude greater than that of a FIB or even plasma FIB (PFIB). With increasing trends towards large volume analysis (LVA), there exists an opportunity for fs-lasers. The combination of LVA needs and the athermal nature of fs-laser machining drove the creation of a TriBeam system comprising a $\mathrm{SEM} / \mathrm{PFIB} /$ fs-laser [1-2] primarily used for LVA. But more recent studies have demonstrated that in situ fs-laser machining offers some unanticipated advantages on many of the beam-sensitive materials described previously.

Here we present results of fs-laser processing of a variety of delicate materials in a TriBeam system. We are focused less on the LVA capabilities, and more on emphasizing its application to these beam-sensitive materials. In Figure 1, we show that fs-laser serial section tomography (SST) can be used to rapidly address a damage susceptible and highly charging resin-embedded mouse brain. Laser cut quality is adequate to see enough cellular detail to make an informed decision about a sub-region to target with higher resolution. The cellular region of interest is then sectioned further with reactive oxygen PFIB [3] to yield either a high resolution tomogram or cross-section. Figure 2 is a collage of fs-laser results on extremely challenging materials that include a multilayer organic light emitting diode (OLED) display, polymers, ultrahard and electrically insulating smartphone glass, and a carbon fiber/epoxy composite (CFEC). These materials may be very difficult to FIB process due to low milling rates, charging, delamination, physiochemical damage, etc. Performing fs-laser pre-processing allows the user to minimize FIB dose and current density requirements for targeted analysis of ion-sensitive samples. In some cases, the fs-laser enables in situ processing of materials that cannot be addressed by FIB.

References:

[1] SJ Randolph et al., Journal of Vacuum Science \& Technology B36 (2018), p. 06JB01. 
[2] MP Echlin and A Mottura et al., Review of Scientific Instruments 83 (2012), p. 023701.

[3] A Martin at al., Scientific Reports 5 (2015), p. 8958.
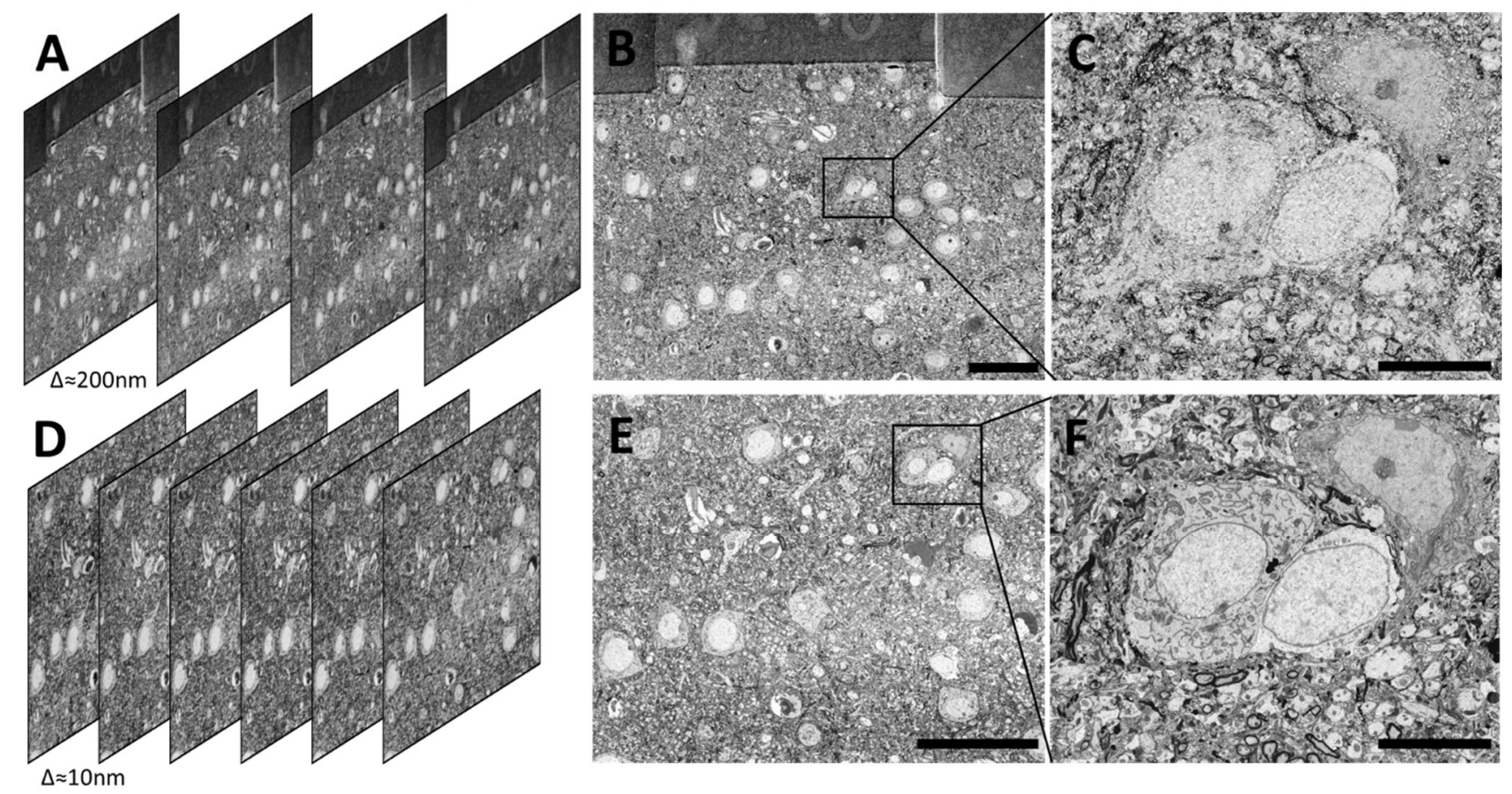

Figure 1. SEM images of resin-embedded mouse brain: (A) Laser serial sections performed until target cell $(B, C)$ was identified. (D) Oxygen PFIB serial sections on target cell with higher resolution and enhanced imaging contrast to produce final images (E,F). Scale bar: $50 \mu \mathrm{m}$ in B,E. $10 \mu \mathrm{m}$ in C, F.
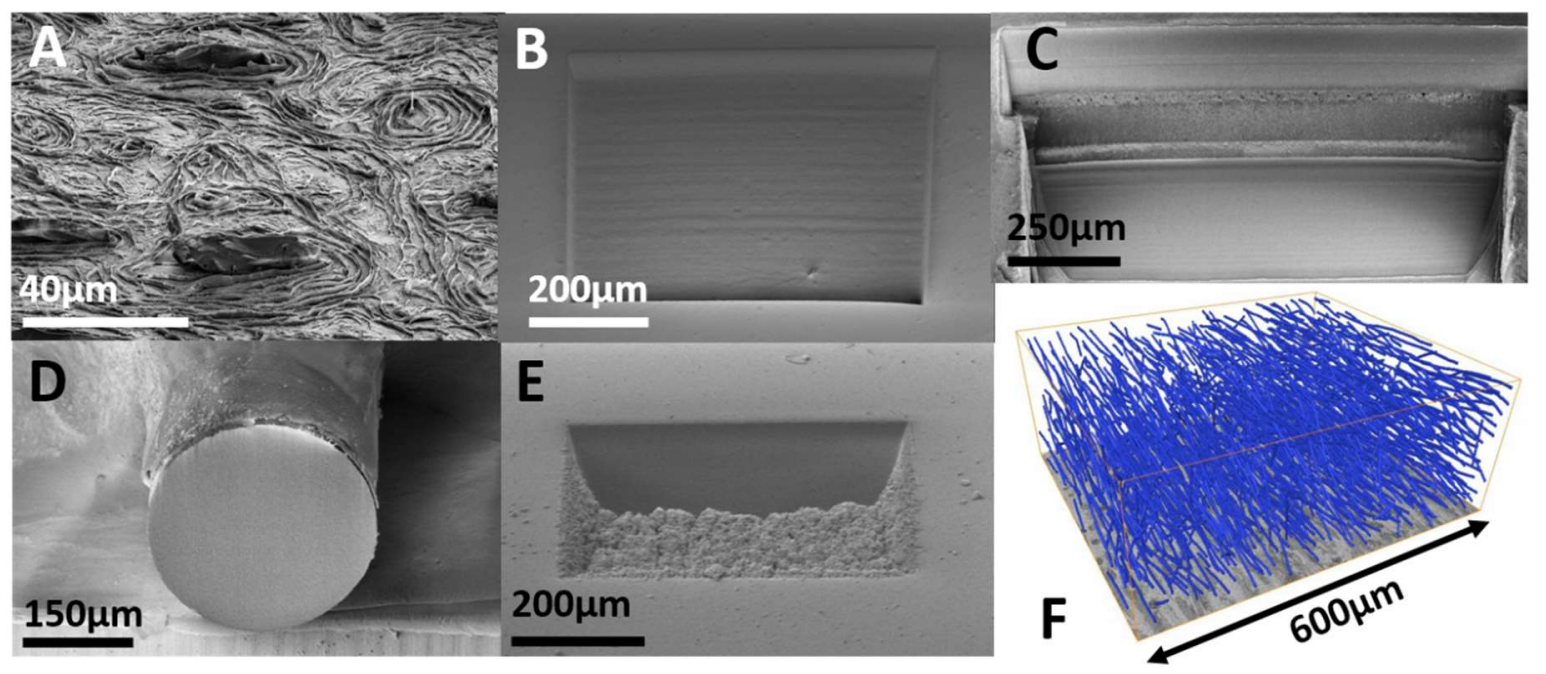

Figure 2. SEM images of polyimide film with excessive PFIB current (A) and as cut by fs-laser (B). SEM of multilayer OLED cross-sectioned by fs-laser (C) showing minimal damage without delamination. SEM images of laser cut nylon fiber (D) and smartphone glass (E) that suffer from melting and low mill rates, respectively, when FIB processed. Differential FIB milling of CFEC results in excessive curtaining artifacts, while fs-laser tomography (laser-SST/backscatter SEM) is less susceptible to differential ablation, thus enabling 3D tomography of these composites $(\mathrm{F})$. 